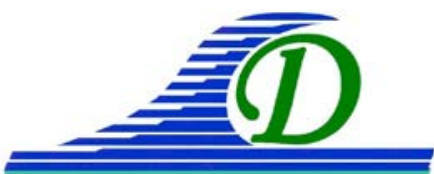

XIII ${ }^{\text {èmes }}$ Journées Nationales Génie Côtier - Génie Civil Dunkerque, 2-4 juillet 2014

DOI:10.5150/jngcgc.2014.117 ～(C) Editions Paralia CFL

disponible en ligne - http://www.paralia.fr - available online

\title{
L’emploi de sédiments traités pour la construction d'une digue de compartimentation (Belgique)
}

\section{Sofie VAN ZELE ${ }^{1}$, Ivo PALLEMANS ${ }^{1}$, Joris VAN NEDERKASSEL ${ }^{1}$, Tom VERMEERSCH ${ }^{2}$, Hans QUAEYHAEGENS ${ }^{3}$}

\section{Envisan NV/SA, membre du Jan De Nul Group, Tragel 60, 9308 Aalst, Belgique. info@envisan.com}

2. Jan De Nul Group, Tragel 60, 9308 Aalst, Belgique. info@jandenul.com

3. Waterwegen \& Zeekanaal NV/SA - département Escaut maritime, Lange Kievitstraat 111-113, 2018 Anvers, Belgique. zeeschelde@wenz.be

\section{Résumé :}

Après les inondations de 1953 et 1976, causées par la Mer du Nord, un plan antiinondation baptisé "plan Sigma" a été élaboré en vue de protéger, de futures marées de tempêtes, la partie flamande de l'estuaire de l'Escaut. Ce plan est régi par Waterwegen en Zeekanaal NV (Voies Navigables et Canal Maritime - W\&Z), une agence publique flamande, qui entend depuis 2005 combiner la protection contre les inondations à la réhabilitation de l'écologie du fleuve par la création de zones d'inondation contrôlées. Les sédiments dragués des voies navigables présentant une qualité similaire sont généralement déshydratés dans des centres de traitement spécialisés, soit par lagunage, soit par déshydratation naturelle. Ces méthodes nécessitent non seulement du temps, mais aussi de vastes espaces et également un investissement financier pour l'aménagement de bassins de déshydratation.

W\&Z a lancé, à Dendermonde, un projet pilote comprenant la construction d'une digue de compartimentation dans une zone d'inondation contrôlée en utilisant des matériaux dragués provenant de l'Escaut, et ce, sans stockage intermédiaire entre les phases de dragage et de construction.

Comme c'est le cas dans de nombreux cours d'eau, le matériau à draguer ne possède pas les caractéristiques géotechniques requises, en raison de la finesse de sa granulométrie, pour être employé directement en construction. C'est pourquoi Jan De Nul Group ainsi que sa filiale dédiée à l'environnement, Envisan, ont organisé une vaste campagne d'essais, en vue de mettre au point un sédiment traité pouvant être employé lors de la phase de construction. Au cours de cette campagne, les effets de plus de 250 combinaisons d'additifs spécifiques sur les caractéristiques géotechniques du matériau dragué ont été analysés. Sur la base des résultats, du modèle géotechnique de digue ainsi que d'une évaluation économique, l'une de ces combinaisons a été choisie pour être utilisée à grande échelle.

Une fois dragué par voie mécanique et transporté par barges vers un lieu proche des travaux de protection anti-inondation, les sédiments ont été pompés à terre dans un 


\section{Thème 8 - Gestion et valorisation des sédiments marins}

centre de stabilisation sur environ 600 m sans liquéfier les sédiments avec de l'eau. Ce centre de stabilisation, construit sur mesure, contrôle en permanence le flux entrant de sédiments et dose automatiquement les additifs afin d'obtenir un matériau stable pour la construction de la digue.

Un programme Assurance Qualité/Contrôle Qualité (QA/QC) a été mis en place pour s'assurer que les performances de la digue soient effectivement celles prévues lors des calculs de conception.

Ce projet, exécuté dans le cadre du projet européen PRISMA (Promoting Integrated Sediment Management - Promotion de la gestion intégrée des sédiments), a prouvé que les sédiments issus du dragage d'entretien, généralement considérés comme inaptes pour tout réemploi utile, peuvent être transformés en matériau de construction de valeur économiquement intéressant pour la réalisation de digues.

Mots-clés : Réemploi utile des sédiments, Solidification, Stabilisation, Construction de zones d’inondation contrôlées, Respect de la nature.

\section{Introduction}

1.1 Mission de Waterwegen \& Zeekanaal à l'égard du réemploi de sédiments dragués Waterwegen en Zeekanaal (Voies Navigables et Canal Maritime - W\&Z) est une agence publique responsable de 1000 kilomètres de voies navigables en Flandre, Belgique, et de la plupart des berges attenantes. La proximité de la Mer du Nord présente par ailleurs un risque d'inondation potentielle. Après les terribles inondations de 1953 et 1976 de la Mer du Nord, un plan anti-inondation baptisé "plan Sigma" a été élaboré en vue de préserver la partie flamande de l'estuaire de l'Escaut de futures marées de tempête. La mise en œuvre de ce plan Sigma est l'une des principales missions de W\&Z. Depuis 2005, la protection anti-inondation est combinée à la réhabilitation de l'écologie du fleuve par la création de zones d’inondation contrôlées. Or, environ 2,5 millions de $\mathrm{m}^{3}$ de matériaux de construction sont nécessaires pour construire de nouveaux remblais autour de la première série de zones d'inondation contrôlées.

W\&Z a pour autre mission majeure le maintien de la navigabilité des voies navigables intérieures. Or, le dragage d'entretien produit des quantités considérables de sédiments. Selon l'analyse annuelle de la qualité des sédiments prélevés dans l'estuaire de l'Escaut, au moins $80 \%$ de ceux-ci sont aptes, sur le plan chimique, à un réemploi dans des travaux d'infrastructure. Cependant, en pratique, la médiocrité de leur qualité géotechnique est souvent considérée comme un obstacle, si bien que de grandes quantités de matériaux dragués sont toujours évacuées dans des décharges contrôlées, ce qui entraîne des coûts importants et soulève souvent une opposition sociétale. Pour éviter ceci, il faut approfondir les connaissances relatives aux techniques permettant un réemploi utile des sédiments. L’impact sur l'environnement des travaux de dragage et de grands travaux d'infrastructure tels que la construction de zones d'inondation 


\section{XIII ${ }^{\text {èmes }}$ Journées Nationales Génie Côtier - Génie Civil \\ Dunkerque, 2-4 juillet 2014}

contrôlée peut être considérablement réduit si ces deux activités sont combinées. Le projet pilote décrit ci-après a été réalisé dans le cadre du partenariat européen PRISMA (Promoting Integrated Sediment Management - Promotion de la gestion intégrée des sédiments).

\section{2 Élaboration du projet pilote de Vlassenbroek}

W\&Z a décidé de lancer un projet pilote global comprenant simultanément la réalisation d'un dragage d'entretien et la construction d'une digue impliquant le réemploi à 100\% des sédiments dragués à Vlassenbroek. La digue de compartimentation prévue à l'intérieur de la future zone d'inondation contrôlée de Vlassenbroek servira de séparation entre une zone de marée présentant une marée contrôlée d’ampleur réduite et une zone uniquement inondée en cas de marée de tempête (voir la figure 1).

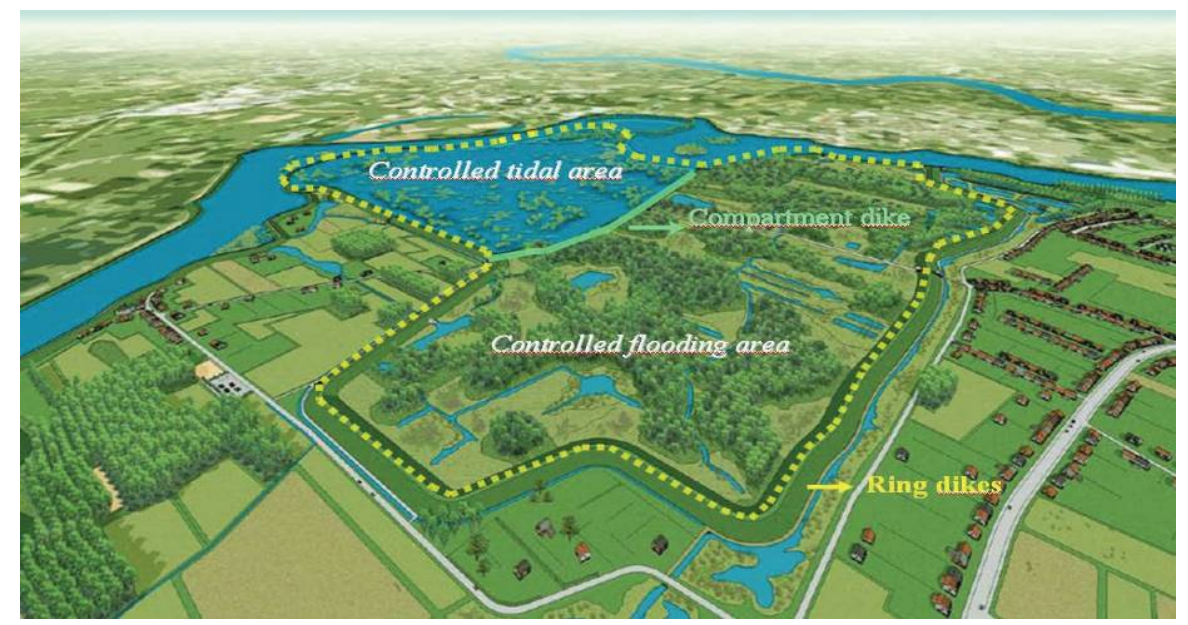

Figure 1. État final de la zone d'inondation contrôlée de Vlassenbroek.

Afin d'encourager l'esprit d'innovation sur le marché, W\&Z a établi un projet de conception et de construction par le biais d'une adjudication ouverte. Pour sélectionner l'offre la plus avantageuse d'un point de vue économique, lors de la procédure d'adjudication, non seulement le prix, mais aussi, la quantité de matériau dragué réemployé ainsi que la qualité technique et environnementale de l'offre ont été étudiés. Il a été demandé aux soumissionnaires de développer et de mettre en œuvre une méthode pour draguer, transporter et réutiliser le matériau dragué dans la construction de la digue de compartimentation située à l'intérieur de la future zone d’inondation contrôlée de Vlassenbroek, et ce, en respectant des conditions strictes au niveau de la délimitation. Des prescriptions ont été imposées au niveau de la forme de la digue de compartimentation, ainsi que des exigences minimales en matière d'imperméabilité et de micro-/macrostabilité. Ces paramètres tiennent compte des conditions de marée à l'endroit précis de la digue de compartimentation dans l'estuaire de l'Escaut. Pour garantir un réemploi significatif des sédiments, l’utilisation de matériau dragué pour la 


\section{Thème 8 - Gestion et valorisation des sédiments marins}

construction d'au moins $60 \%$ du volume total de la digue de compartimentation, estimé à $100000 \mathrm{~m}^{3}$, a été imposée. Une fois dragué, le matériau devait être transporté soit par voie navigable, soit via des pipelines, jusqu'au site de construction, pour y être employé sur-le-champ. Tout stockage intermédiaire était exclu afin de minimiser l'impact des travaux de construction sur l'environnement.

\section{Phase de conception}

\section{1 Étude du site}

\subsubsection{Caractérisation des sédiments}

Une vaste campagne d'échantillonnage des sédiments a été réalisée le long de la portion à draguer de l'Escaut, longue de 2,5 kilomètres, et ce à l'aide d'une carotteuse à piston. Conformément à la législation flamande (Vlarema), relative au réemploi des déchets, les sédiments non pollués peuvent donc être réutilisés comme matériau de construction. D’un point de vue géotechnique, les sédiments présentent une texture de limon sableux fin ne possédant pas les caractéristiques géotechniques nécessaires pour une application dans des travaux d'infrastructure sans traitement.

\subsubsection{Caractérisation géotechnique du sous-sol}

Pour bien comprendre les caractéristiques géotechniques du matériau appliqué dans le site de construction, il est crucial d'effectuer une identification détaillée du sous-sol. À cette fin, de nombreux sondages ont été forés jusqu'à une profondeur de $20 \mathrm{~m}$ dans le sous-sol. À chaque endroit ont été prélevés au moins cinq échantillons intacts, uniformément répartis sur le profil sondé. Parmi les essais et analyses effectués sur ces échantillons, nous pouvons citer : répartition de la taille des particules, consistance du sol/taux d'eau, contenu organique, paramètres de tassement et de consolidation et détermination des paramètres de résistance au cisaillement. Ces données ont été complétées par des essais sur le terrain comprenant un test de pénétration au cône (CPT) et des tests au moyen d'un scissomètre de poche.

Le sous-sol en question est composé des couches suivantes, en partant du niveau du sol : argiles plastiques meubles interrompues par une couche de tourbe saturée, couche de sable argileux, puis de l'argile dure du Tertiaire par-dessus une couche de sable. Sous la couche de sable, on note la présence d'une couche d'argile dure reposant sur un dépôt de sable.

Les données tirées de la caractérisation géotechnique des sédiments et du sous-sol ont été intégrées aux calculs de conception géotechnique réalisés avec le logiciel SLOPE/W. 


\section{XIII ${ }^{\text {èmes }}$ Journées Nationales Génie Côtier - Génie Civil \\ Dunkerque, 2-4 juillet 2014}

\subsection{Critères de conception}

La digue de compartimentation à construire avait une longueur de 800 mètres, des pentes de 20/4 et une largeur de 7 mètres en crête, pour une hauteur de 4,5 mètres audessus du sol. Son volume était d'environ $100000 \mathrm{~m}^{3}$.

Les critères de conception étaient semblables à ceux d'une digue traditionnelle soumise aux marées. Des mécanismes de défaillance tels que percement, éclatement et retassure devaient être abordés selon des facteurs de sécurité donnés. De plus, des instabilités telles que le soulèvement de couches imperméables et la formation de plans de glissement, ou encore l'influence des niveaux de marées de tempête sur la stabilité, devaient être vérifiées.

La perméabilité du matériau de la digue devait être inférieure à $1,0 \times 10^{-7} \mathrm{~m} / \mathrm{s}$.

Enfin, les éventuels tassements devaient être pris en compte dans la conception de la digue.

\subsection{Solution adoptée}

Généralement, les sédiments fluviaux, de qualité analogue, dragués sont déshydratés dans des centres de traitement spécialisés, soit par lagunage, soit par déshydratation naturelle. Le lagunage s'effectue dans des bassins imperméables dotés d'un système de drainage. Ces bassins peuvent être utilisés plusieurs fois, mais le coût d'investissement est élevé. En outre, il faut entre 3 et 9 mois pour que le lagunage soit achevé ; c'est pourquoi cette méthode n’a pas été retenue comme solution réalisable.

Les sédiments dragués peuvent aussi être déshydratés au moyen de géotubes. Compte tenu du retour d'expérience de Jan De Nul Group, cette technique révèle certains inconvénients, comme le besoin d'un volume important d'eau de transport, la difficulté d'évaluer les tassements et le risque que l'eau emprunte des voies de prédilection entre les éléments de géotube.

La technique consistant à solidifier les terres meubles par des additifs tels que de la chaux est largement répandue en construction civile. En Scandinavie particulièrement, il n'est pas rare que des sédiments pollués soient solidifiés à l'aide d'additifs avant d'être définitivement évacués dans une décharge contrôlée (SMOCS, 2012; SWEDISH GEOTECHNICAL INSTITUTE, 2006 ; CASST, 2004). L’usage de sédiments solidifiés présente les avantages suivants :

- Aucune eau de transport, ni permis environnementaux associés, requis ;

- Le corps de la digue peut être bâti en employant une proportion élevée de sédiments, ce qui réduit les fournitures externes ;

- L’ouvrabilité des sédiments est rapidement améliorable ;

- La portance est rapidement améliorable ;

- Risque minimum de glissements internes, grâce à l'usage d'un seul matériau ;

- Réduction des tassements puisqu'aucune déshydratation ne survient et que la résistance finale est atteinte au bout d'à peine deux semaines. 


\section{Thème 8 - Gestion et valorisation des sédiments marins}

Sur la base de cette analyse des technologies, Jan De Nul Group a choisi la solution novatrice des sédiments traités, fondée sur la technique de la solidification.

\subsubsection{Calculs de conception}

\subsubsection{Perméabilité de la digue et stabilité de la pente.}

Les calculs de stabilité ont été réalisés à l'aide du logiciel SLOPE/W, et le modèle de filtration au moyen de SEEP/W.

Les paramètres encodés provenaient d'études de laboratoire et sur site. Selon les tests de laboratoire effectués sur des sédiments traités, les caractéristiques minimales du matériau solidifié sont les suivantes :

- Perméabilité à l'eau (kf) : 1,0×10 $\mathrm{m} / \mathrm{s}$ (ISO 17892-11)

- Résistance au cisaillement non drainé (cu) : 35 kPa (BS 1377-7)

- Angle de frottement interne $(\varphi): 25^{\circ}$ (BS 1377-8)

- Cohésion (c) : 4 kPa (BS 1377-8)

Les paramètres du sous-sol demeurant inconnus ont été déterminés en recourant au tableau des Annexes Nationales indiquant les valeurs caractéristiques relatives au sol.

En guise de conditions limite pour le calcul de filtration transitoire, un scénario pessimiste impliquant 7 jours de niveau d'eau en permanence élevé dans la zone de marée contrôlée (équivalent au niveau en crête de la digue de compartimentation) et une zone d'inondation contrôlée vide, a été retenu. Un tableau hydraulique a été modélisé pour ces conditions limites.

Le débit d'eau total calculé dans et sous la digue de compartimentation est de $1,4942 \mathrm{E} \times 10^{-7} \mathrm{~m}^{3} / \mathrm{s} / \mathrm{m}$ digue. Le tableau hydraulique modélisé a en outre servi pour les calculs de stabilité de la pente. On a recouru à la méthode de Bishop pour déterminer la surface de glissement critique. Les phases de construction ont été calculées non drainées, avec un facteur de sécurité global de 1.1, tandis que les états finaux ont été calculés drainés avec un facteur de sécurité global de 1.3.

Une fois la dernière phase de construction (matériau non drainé) achevée, a pu être observé un facteur de sécurité minimum de 2.08. Concernant l'état final, sous les niveaux d'eau les plus élevés, a été obtenu un facteur de sécurité minimum de 1.36 contre les défaillances de glissement de surface dans la zone d'inondation contrôlée, ce qui satisfaisait aux critères.

\subsubsection{Tassements}

Pour calculer les tassements du sous-sol, la méthode de Sanglerat a été utilisée. Les paramètres relatifs au sous-sol ont été extraits des sondages CPT. Le matériau de la digue présentait une densité de $16,5 \mathrm{kN} / \mathrm{m}^{3}$. Le tassement du sous-sol a été estimé à $16 \mathrm{~cm}$. 


\section{XIII ${ }^{\text {èmes }}$ Journées Nationales Génie Côtier - Génie Civil \\ Dunkerque, 2-4 juillet 2014}

\subsubsection{Sédiments traités}

Pour améliorer les propriétés géotechniques des sédiments, on a choisi d'ajouter à ceuxci les additifs adéquats. Les liants hydrauliques et pouzzolaniques font partie du vaste assortiment d'additifs sur le marché. Toutefois, seuls quelques-uns d'entre eux conviennent pour ce projet en raison des contraintes géotechniques et économiques. Plusieurs types et combinaisons d'additifs ont été mélangés aux sédiments et testés dans une étude extensive menée en laboratoire.

\subsubsection{Sélection et dosage des additifs}

La procédure de sélection initiale s'est concentrée sur l'augmentation de la solidité selon quatre paramètres clé (BOUTOUIL, 1999 ; BOUBAKER \& BOUTOUIL, 2009) :

- Type d'additifs ;

- Dosage ;

- Taux d'eau du sédiment ;

- Temps de durcissement.

Dans un premier temps, les additifs ont été passés au crible afin de sélectionner ceux affichant des résultats intéressants. Une campagne d'essais, plus approfondie en vue d'identifier l'(les)additif(s) le plus approprié, a précédé ce criblage. La procédure de suivi des essais ainsi que l'interaction avec la conception de la digue sont représentés à la figure 2 .

Ciments, chaux à prise rapide, cendres volantes, mâchefer, laitier de haut fourneau en granulés et silicate de sodium font partie des additifs testés. Outre les propriétés géotechniques, la présence d'une certification adéquate et du rapport coût/efficacité ont aussi été pris en compte pour choisir l'(les) additif(s). Lors des tests en laboratoire, des échantillons ont été réalisés en malaxant sédiments et additifs jusqu'à l'obtention d'une masse homogène. Ces échantillons ont été stockés dans des récipients en plastique étanches à l'air. Après 7, 14 et 28 jours de durcissement, la résistance de cisaillement non drainé obtenue par les échantillons a été déterminée en ayant recours à un scissomètre motorisé de laboratoire.

Il est ressorti des calculs de conception de la digue que les sédiments traités devaient présenter une résistance de cisaillement non drainé d'un minimum de $35 \mathrm{kPa}$. Il est évident que le taux d'eau et le dosage de l'additif jouent un rôle crucial. 


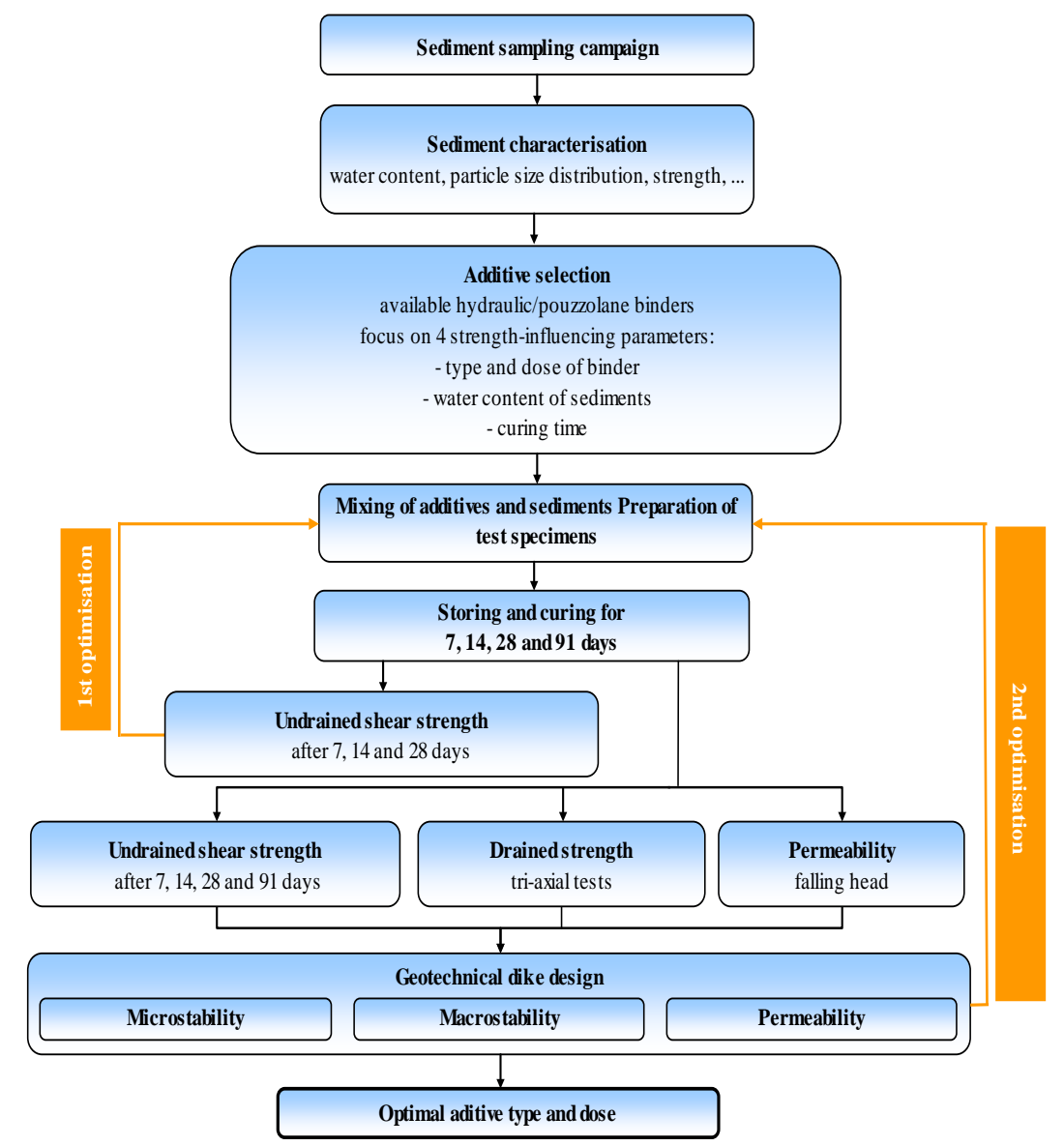

Figure 2. Organigramme montrant la procédure de suivi des essais de labo et l'interaction avec les calculs de conception de la digue.

Sur la base des résultats du criblage, un mélange de ciment de Portland et de cendres volantes à silice hautement réactive a été retenu comme additif le plus performant. Dans une phase ultérieure, le dosage de ce mélange a été optimisé, si bien qu'en deux semaines, les sédiments traités avaient développé une résistance suffisante pour supporter des engins lourds, et il est apparu des calculs de stabilité que la perméabilité devait s'élever au minimum à $1,0 \times 10^{-7} \mathrm{~m} / \mathrm{s}$. On observe un seuil à partir duquel la résistance commence à augmenter de façon exponentielle. Il est ressorti d'un suivi de la résistance effectué jusqu’à 91 jours que 95-99\% de la résistance maximale développée sont obtenus après 28 jours.

Les tests de perméabilité (méthode à niveau variable) ont révélé que la conductivité hydraulique de ces mélanges variait entre $6,3 \times 10^{-9}$ et $3,6 \times 10^{-8} \mathrm{~m} / \mathrm{s}$, ce qui était largement au-dessous de $1,0 \times 10^{-7} \mathrm{~m} / \mathrm{s}$ requis. 


\section{XIII ${ }^{\text {èmes }}$ Journées Nationales Génie Côtier - Génie Civil \\ Dunkerque, 2-4 juillet 2014}

\section{Phase d'exécution}

\subsection{Dragage et construction de la digue}

Les sédiments ont été dragués et chargés dans des barges par voie mécanique. Le dragage a pu être effectué en continu, et ce, malgré une marée d'environ 5 mètres d'amplitude. Les barges ont été amenées à l'aide de bateaux pousseurs jusqu'au lieu de destination. Les barges de transport contenaient en général des chargements entre 300 et $550 \mathrm{~m}^{3}$. Contrairement au dragage hydraulique, le dragage mécanique génère des sédiments près de la densité in situ, puisqu'aucune eau n’est nécessaire pour transférer le matériau du lit du fleuve à la surface de l'eau.

À l'endroit du déchargement, une jetée de déchargement spécialement conçue a été construite, qui prenait en compte l'importante d'amplitude de la marée et des courants forts (jusqu'à 4 nœuds) de l'Escaut à Vlassenbroek. Au bout de cette jetée une plateforme de déchargement flottante a été placée afin de décharger les barges qui arrivaient (voir la 3).

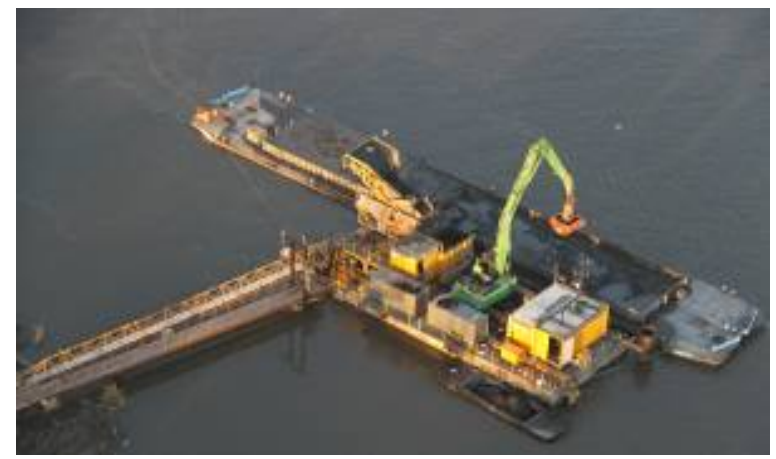

Figure 3. Plateforme de déchargement avec installation de pompage.

Une excavatrice de transbordement longue portée a été utilisée pour décharger le matériau surun tamis vibrant. Le double tamis, pourvu de mailles de $150 \mathrm{~mm}$ et $50 \mathrm{~mm}$ de diamètre, éliminait les macro-déchets et les pierres. Ensuite, le matériau criblé était enfermé dans une trémie munie d'un système de distribution à vis. De là, le matériau haute densité était injecté par des pompes à piston dans le pipeline vers l'unité de traitement des sédiments.

Selon le type de matériau, la capacité globale de déchargement s'est élevée à entre 80 et $130 \mathrm{~m}^{3}$ par heure de service. Chaque pompe était reliée à un pipeline de $580 \mathrm{~m}$ de long et $250 \mathrm{~mm}$ de diamètre, prévu pour résister à des pressions élevées, de maximum 80 bars.

À l'autre extrémité du pipeline, les sédiments arrivaient dans l'unité de stabilisation, une unité de mélange composée de 2 malaxeurs à pales indépendants, dotés chacun de leur propre système de dosage des additifs calibré. Le volume entrant de sédiments a été 


\section{Thème 8 - Gestion et valorisation des sédiments marins}

mesuré en permanence, et le dosage des additifs ajusté à ce flux entrant par le biais de vis de dosage à variateur de fréquence régulièrement calibrées. Une fois le malaxage terminé, le matériau traité stabilisé était envoyé dans un silo de $45 \mathrm{~m}^{3}$ avant d'être chargé dans des tombereaux. Ces derniers transportaient les sédiments traités stabilisés jusqu'au chantier de construction de la digue, où ils étaient directement déchargés sur la berge. Le matériau a alors durci sur place, atteignant la résistance et la dureté voulues pour la digue.

A l'issue du temps de durcissement prévu, une excavatrice légère a nivelé les sédiments traités pour profiler la digue. L'expérience nous a appris que les engins lourds pouvaient travailler sur le matériau après 4 à 5 jours.

\subsection{Contrôle de la qualité}

Un programme Assurance Qualité/Contrôle Qualité (QA/QC) a été mis en place pour assurer la qualité déterminée tout au long du projet. Ce programme comprenait la construction de parcelles test, le contrôle du taux d'eau des sédiments dragués, le test de la qualité du mélange d'additifs ainsi que de la précision du dosage et du malaxage. Sur le plan géotechnique, le programme comprenait la collecte de données sur le terrain et des analyses de la répartition des grains des particules, de la résistance et de la perméabilité.

\subsubsection{Supervision du traitement}

Plus de $100000 \mathrm{~m}^{3}$ de matériau devaient être traités. Les sédiments dragués provenaient d'une section de 2,5 km de long de l'Escaut devenue non navigable dans les années 70 . Afin de déterminer le traitement, l'hétérogénéité du taux d'eau, la granulométrie et le contenu organique du matériau, il a fallu caractériser les matériaux. Par conséquence, la qualité géotechnique des sédiments dragués a été vérifiée à chaque barge.

Ensuite, les paramètres opérationnels du pompage et du malaxage ont pu être ajustés en fonction de la nature des sédiments traités.

\subsubsection{Résistance de la digue}

Un scissomètre de poche a permis de mesurer la résistance au cisaillement non drainé et de vérifier rapidement la résistance des sédiments traités sur le terrain. Pendant les travaux, la résistance a régulièrement été mesurée selon une trame uniforme comprenant des points de mesure tous les $30 \mathrm{~m}$. À chaque point de la trame, des mesures étaient effectuées à la surface, et à chaque mètre, verticalement. Les résultats obtenus par le scissomètre de poche ont été corrigés en fonction de la plasticité, l'anisotropie et les effets de la vitesse, et ce, selon la formule de Bjerrum (VAN 't HOFF \& VAN DER KOLFF, 2012).

D’après les résultats des essais de résistance effectués sur site, la résistance moyenne au cisaillement non drainé était de $76 \pm 29 \mathrm{kPa}$ à la surface et de $55 \pm 20 \mathrm{kPa}$ en 


\section{XIII ${ }^{\text {èmes }}$ Journées Nationales Génie Côtier - Génie Civil \\ Dunkerque, 2-4 juillet 2014}

profondeur. Le développement d'une plus grande résistance à la surface s'explique par le séchage et le compactage naturels du matériau dus aux engins de terrassement.

Après l'essai sur le terrain, des échantillons non remaniés ont été prélevés afin de déterminer la cohésion et l'angle de friction via un test triaxial en laboratoire (BS 13778, contrepression $300 \mathrm{kPa}$, pressions de consolidation $\sigma 350,75$ et $100 \mathrm{kPa}$ ). L'angle de friction mesuré variait entre 34,0 et $40,0^{\circ}$, et la cohésion entre 8,4 et $34,2 \mathrm{kPa}$.

Les résultats sur le terrain ont révélé que les paramètres de résistance des sédiments traités étaient nettement supérieurs aux paramètres minimum requis en vertu des calculs de conception (voir section 0).

\subsubsection{Imperméabilité de la digue}

Un infiltromètre à double anneau (voir figure 4), ASTM D3385-03, a été utilisé pour mesurer la conductivité hydraulique et pour vérifier rapidement la perméabilité verticale à l'eau de surface des sédiments traités sur le terrain.

La perméabilité était comprise dans une fourchette de $1,0 \times 10^{-8}$ à $8,3 \times 10^{-8} \mathrm{~m} / \mathrm{s}$. Après l'essai sur le terrain, des échantillons non remaniés ont été prélevés afin de valider les mesures sur le terrain par une mesure de la perméabilité en laboratoire (NBN ISO 17982-11, tension d'élément de $300 \mathrm{kPa}$ et charge de $50 \mathrm{kPa}$ ). Il en a résulté des perméabilités de $1,6 \times 10^{-9}$ à $3,4 \times 10^{-8} \mathrm{~m} / \mathrm{s}$.

Les résultats sur le terrain ont révélé que la perméabilité des sédiments traités vérifiés était largement en-deçà de la perméabilité maximum admise dans les calculs de conception (voir section 0).

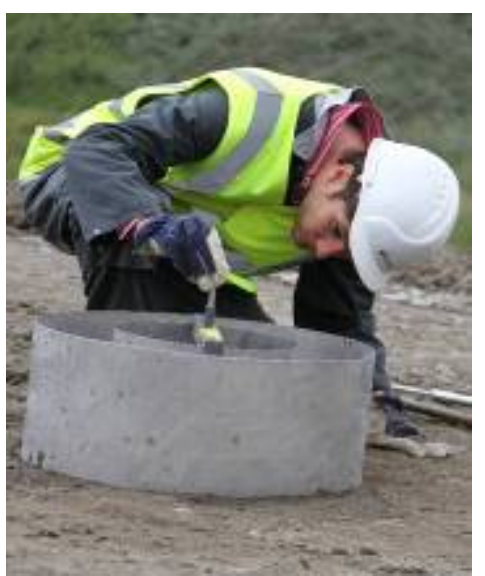

Figure 4. Mesure de la perméabilité sur le terrain au moyen d'un infiltromètre à double anneau.

\subsection{Couverture végétale}

Il a été décidé de poser une couverture végétale sur les pentes de la digue afin de protéger ses surfaces construites de l'érosion. 


\section{Thème 8 - Gestion et valorisation des sédiments marins}

Il a été conclu d'une tentative d'ensemencement pré-test qu'une méthode de semis classique (labourage, ensemencement et passage au rouleau) n'entraînerait pas une pousse rapide des grains d'herbe, car le temps sec et les caractéristiques défavorables du sous-sol (faible perméabilité, haute alcalinité) empêchaient la germination.

Par conséquent, les près de $35000 \mathrm{~m}^{2}$ de pente de la digue ont été ensemencés par voie hydraulique. Le mélange de semences ( $50 \%$ de fétuque rouge, $50 \%$ de fétuque ovine), dosé à $300 \mathrm{~kg} / \mathrm{ha}$, a été mélangé à de l'eau, du mulch et des liants, le liant à base d'amidon permettant à la semence d'adhérer aux pentes de la digue, un autre polymère faisant office d'agent de rétention d'eau et le mulch améliorant la fertilité de la surface. Pendant les deux semaines suivant l'ensemencement, les pentes de la digue ont été arrosées tous les 4 jours, si bien qu'au bout de deux semaines, la germination des graine a pu être observée.

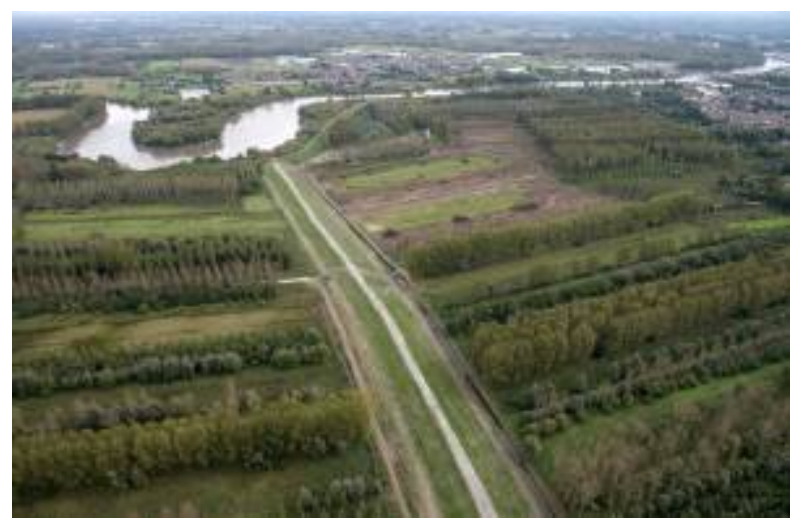

Figure 5. Digue de compartimentation achevée - automne 2013.

\section{Phase ultérieure à la construction}

Trois mois après l'achèvement de la construction de la digue, une campagne d'essais visant à vérifier l'homogénéité du corps de la digue a été lancée. Ce programme a inclus un test de pénétration au cône (CPT), des tests en laboratoire (essai scissométrique en laboratoire, essai triaxial, œdomètre, granulométrie) et un test de perméabilité sur le terrain réalisé au moyen du perméamètre de Boutwell.

À la date de rédaction de ce document, seuls les résultats du test de pénétration au cône sont connus.

Le test de pénétration au cône est un bon moyen de vérifier sur le terrain l'homogénéité du matériau de la digue. Cet essai a l'avantage de fournir un profil continu immédiat. Les sondages ont été réalisés à l'aide d'un pénétromètre de $200 \mathrm{kN}$ monté sur chenilles. La résistance de pointe $(q c)$ et la friction de pointe ( $f s)$ ont été enregistrées tous les $2 \mathrm{~cm}$ en 16 endroits répartis sur toute la digue.

Les profils CPT relevés en ces 16 points ont montré une similarité satisfaisante. La couche supérieure de la digue présente une résistance élevée, car une route dotée d’une 


\section{XIII ${ }^{\text {èmes }}$ Journées Nationales Génie Côtier - Génie Civil \\ Dunkerque, 2-4 juillet 2014}

couche de 0,5 m de gravier a été aménagée sur la crête de la digue. Ensuite, la résistance de pointe tombe à $800 \mathrm{kPa}$ et reste quasi inchangée sur 4 à 5 mètres, ce qui indique une bonne homogénéité de la couche de sédiments traités.

L'augmentation de la résistance aux environs de 5 mètres est liée au sous-sol sur lequel la digue de compartimentation a été construite.

On peut déduire, le calcul au cisaillement non drainé permet de déterminer la résistance de la pointe du cône (BURGOS et al., 2007 ; VAN 't HOFF \& VAN DER KOLFF, 2012). La formule souvent utilisée pour déduire $c u$ d'un CPT est :

$c u=\frac{q c}{N c}$

Où $N c$ est un facteur empirique généralement compris entre 10 et 13 . Ce rapport doit être calibré par des essais scissométriques sur des échantillons non remaniés prélevés au cours de la campagne d'essais ultérieure à la phase de construction.

\section{Conclusion}

L’agence publique flamande Waterwegen \& Zeekanaal a favorisé le réemploi utile des sédiments en encourageant l'utilisation de sédiments provenant de dragages d'entretien en tant que matériau de construction d'une digue dans la zone d'inondation de Vlassenbroek. Dans leur forme naturelle draguée, ces sédiments ne satisfaisaient pas aux critères géotechniques requis. Après avoir étudié de manière approfondie la zone de construction et les sédiments devant être utilisés, Jan De Nul Group a pu mettre au point une procédure innovante pour transformer les sédiments non conformes en matériau de construction de valeur.

Des tests en laboratoire suivis par des essais sur site à grande échelle ont démontré que les critères imposés en matière de stabilité et de perméabilité pouvaient être atteints. En ayant recours à des pompes à haut débit et à une unité de traitement dûment contrôlée, le besoin de retraitement et le stockage intermédiaire du matériau solidifié ont pu être éliminés.

La technique appliquée ouvre la voie à une nouvelle approche de l'emploi des sédiments fins dans les travaux d'infrastructure de grande ampleur. En sélectionnant soigneusement les additifs et le dosage à appliquer, on peut transformer en quelques jours des sédiments inappropriés en matériau de construction utile, avec un minimum de manipulations et un rapport coût/efficacité intéressant.

\section{Références}

BOUBAKER R., BOUTOUIL M. (2009). Organic matter and cement content effect of geotechnical properties of cement treated sediments. Conférence I2SM, Lille, France.

BOUTOUIL M. (1999). Traitement des vases de dragage par solidification/stabilisation à base de ciment et additifs. Thèse de Doctorat, Université de Caen, France, 276 p. 
Thème 8 - Gestion et valorisation des sédiments marins

BURGOS M., SAMPER F., ALONSO J.J. (2007). Improvements carried out in very soft dredged mud soil in the Port of Valencia (Spain). European Conference on Soil Mechanics and Geotechnical Engineering, pp 2091-2104.

CASST (2004). Review of scientific literature on the use of stabilisation/solidification for the treatment of contaminated soil, solid waste and sludges. Disponible en ligne sur: http://www.environment-agency.gov.uk

SMOCS (2012). Report on sustainable management of Contaminated Sediments: Review of Potential Applications for s/s. Disponible en ligne sur : http://www.smocs.eu SWEDISH GEOTECHNICAL INSTITUTE (2006). Report 72 on Strength of stabilised soils - A laboratory study on clays and organic soils stabilised with different types of binder.

VAN 't HOFF J., VAN DER KOLFF A.N. (2012). Hydraulic Fill Manual: For Dredging and Reclamation Works. $1^{\text {st }}$ ed. CRC press. 642 p. 